\title{
Supershear bursts in the propagation of a tensile crack in linear elastic material
}

\author{
Fabian Barras, ${ }^{1, *}$ René Carpaij, ${ }^{1,2}$ Philippe H. Geubelle, ${ }^{2}$ and Jean-François Molinari ${ }^{1}$ \\ ${ }^{1}$ Civil Engineering Institute, Institute of Materials Science and Engineering, \\ École Polytechnique Fédérale de Lausanne (EPFL), Station 18, 1015 Lausanne, Switzerland \\ ${ }^{2}$ Department of Aerospace Engineering, University of Illinois at Urbana-Champaign, 306 Talbot Laboratory, \\ 104 South Wright Street, Urbana, Illinois 61801, USA
}

(Received 11 May 2018; revised manuscript received 13 September 2018; published 7 December 2018)

\begin{abstract}
Since the early years of the linear elastic theory of fracture [linear elastic fracture mechanics (LEFM)], scientists have sought to understand and predict how fast cracks grow in a material or slip fronts propagate along faults. While shear cracks can travel faster than the shear wave speed, the Rayleigh wave speed is the limiting speed theoretically predicted for tensile failure. This work uncovers the existence of supershear episodes in the tensile (mode I) rupture of linearly elastic materials beyond the maximum allowable (sub-Rayleigh) speed predicted by the classical theory of dynamic fracture. While the admissible rupture speeds predicted by LEFM are verified for smooth crack fronts, we present numerically how a supershear burst can emerge from a discontinuity in crack front curvature. Using a spectral formulation of the three-dimensional elastodynamic equations coupled with a cohesive model of fracture, we study how these short-lived bursts create shock waves persisting far from the discontinuity site. This study provides insight on crack front instabilities present in the rapid tensile failure of brittle materials due to large distortions of the rupture front.
\end{abstract}

DOI: 10.1103/PhysRevE.98.063002

\section{INTRODUCTION}

How fast cracks can propagate in linearly elastic solids is a long-standing question that has challenged scientists and engineers for many decades. The classical theory of linear elastic fracture mechanics (LEFM) [1,2] predicts that the Rayleigh wave speed $c_{R}$ of the surrounding bulk material is the limiting propagation speed of tensile (mode I) cracks, while intersonic crack speeds (i.e., between the shear wave speed $c_{s}$ and the dilatational wave speed $c_{d}$ ) are allowed for in-plane shear (mode II) cracks. Using a nonsingular cohesivelike description of fracture, Burridge [3] and Andrews [4] proposed a mechanism enabling shear cracks to transition between the subsonic and intersonic regimes.

Due to crack kinking or branching, the experimentally measured speeds of tensile cracks are substantially lower, rarely exceeding $0.65 c_{R}$ [5-7]. The postmortem observation of the fracture surfaces revealed the origin of the discrepancy between the dynamic fracture theory and the experiments. As the rupture speed increases, the crack stops having a unique sharp tip and becomes a smeared rupture front interplaying with the material microstructure. A complete review of the dynamic fracture experiments and discussions about their inherent instabilities can be found in [5,6,8-11]. The appearance of the fracture surface typically follows a three-stage (mirrormist-hackle) transition. The mirrorlike surface observed at low rupture velocities progressively roughens with the appearance of in-plane surface marks as the crack speed approaches a few tenths of $c_{R}$ (mist). As the crack speed typically exceeds

*Corresponding author. fabian.barras@epfl.ch
$0.4 c_{R}$, aborted microscopic branches start growing out of the rupture plane with a repetitive pattern (hackle), up to the occurrence of a macroscopic branching event if the rupture speed keeps increasing. This progressive onset of dynamic instabilities, which were observed within various kinds of brittle materials $[9,12-15]$, can be suppressed by constraining crack growth along a weaker rupture plane $[16,17]$ or by reducing the specimen thickness up to plane stress conditions [18-20]. The latter revealed the three-dimensional nature of these dynamic instabilities, which occur when the translational invariance is broken, and explained why their theoretical and numerical description is particularly challenging and still overlooked.

The first-order perturbation analyses of an initially straight crack front became well-established approaches to describe the three-dimensional (3D) effects associated with the distortion of quasistatic [21,22] and dynamic crack fronts [23]. These approaches, grounded on the hypothesis of small distortions of the front, have indeed been extensively used to study crack propagation within disordered materials $[24,25]$. However, during dynamic instabilities, large distortions are expected to arise locally along the front, including the formation of cusps [26]. The higher-order effects associated with these large distortions of the crack front are the focus of the present study.

Using a spectral formulation of the 3D elastodynamic equations coupled to a cohesive zone model of fracture, this work investigates the rupture dynamics subsequent to large distortions of the crack front in the presence of a tougher asperity. Our study uncovers how supershear events can also occur during mode I tensile ruptures, and how these events are triggered by local discontinuities emerging along the crack front. 
Supershear fronts and their strong impact on the rupture dynamics received much attention in the context of shear cracks and earthquakes, for which slip fronts have been measured to propagate faster than $c_{s}$ along some portion of the fault [27-31]. Similarly to supersonic aircraft, elastic waves radiated from supershear cracks gather to form shock wave fronts, also referred to as Mach cones, leading to particularly violent earthquakes [32-36]. Several studies have described how local variation in toughness or elastic properties can precisely favor the supershear transition of a mode II crack [37-43]. Despite the relative rarity of supershear earthquakes reported in nature, recent experiments [44] suggest that shortlived supershear events may frequently occur at small scales of crustal fault, out of the resolution of seismic inversion, yet significantly impacting the rupture dynamics.

Similarly, our study demonstrates how the short-lived supershear bursts existing only at the cusp locations along a tensile crack front create nevertheless persistent shock waves, capable of perturbing the dynamics far from the initial discontinuity site. This new result finds direct relevance in the fast tensile rupture of materials characterized by dynamic instabilities, for which crack front discontinuities are expected to occur at heterogeneities $[8,13,14]$ or to emerge from microbranching instabilities [26,45].

\section{SETUP}

We study the in-plane crack front propagation along a weak plane located along $y=0$ in an infinite linearly elastic solid initially at rest under a uniform tensile stress $\sigma_{0}$. At time $t=0$, a straight crack front parallel to the $z$ axis starts to grow dynamically in the positive $x$ direction before encountering a row of asperities. This $3 \mathrm{D}$ fracture problem is solved using a boundary integral formulation of the elastodynamic equations $[46,47]$ relating the normal displacement jump (or crack opening displacement) $\delta_{n}(x, z, t)$ and the normal traction stress $\tau_{n}(x, z, t)$ acting on the fracture plane. This numerical method, referred to as the spectral scheme, allows for a very fine discretization of the fracture plane, especially within the failure zone described by a linear cohesive failure law modeling the evolution of the cohesive strength of the material in the immediate vicinity of the crack front:

$\tau^{\mathrm{str}}(x, z, t)=\tau_{c}(x, z) \max \left[1-\delta_{n}(x, z, t) / \delta_{c}(x, z) ; 0\right]$.

In Eq. (1), $\tau_{c}$ and $\delta_{c}$ respectively denote the spatially varying failure strength and critical crack opening displacement of the weak plane. The nonlinear dissipative processes associated with the dynamic failure event are thus confined to the crack plane, with the fracture energy given by $G_{c}(x, z)=$ $\frac{1}{2} \tau_{c}(x, z) \delta_{c}(x, z)$.

\section{ELASTODYNAMICS AND NUMERICAL METHOD}

Let us consider two semi-infinite solids with the same linearly elastic properties bonded together along the plane $(y=0)$ initially at rest under a uniform tensile stress $\sigma_{0}$ such that

$$
\begin{aligned}
\dot{\boldsymbol{u}}^{+}(x, y, z, t=0) & =\dot{\boldsymbol{u}}^{-}(x, y, z, t=0)=0, \\
\boldsymbol{\sigma}_{y y}^{+}(x, y, z, t=0) & =\boldsymbol{\sigma}_{y y}^{-}(x, y, z, t=0)=\sigma_{0} .
\end{aligned}
$$

In the above equations, $\dot{\boldsymbol{u}}$ is the velocity field and $\sigma$ the Cauchy stress tensor, while the superscripts $+/-$ respectively denote the top $(y>0)$ and bottom $(y<0)$ half spaces. The momentum balance equation in the linear elastic materials in the absence of body forces can be written as

$$
c_{d}^{2} \nabla\left(\nabla \cdot \boldsymbol{u}^{ \pm}\right)-c_{s}^{2} \nabla \times\left(\nabla \times \boldsymbol{u}^{ \pm}\right)=\frac{\partial^{2} \boldsymbol{u}^{ \pm}}{\partial t^{2}},
$$

where $c_{s}$ denotes the shear wave speed, $c_{d}=\sqrt{\frac{2(1-v)}{1-2 v}} c_{s}=\eta c_{s}$ is the dilatational wave speed, and $v$ is the Poisson's ratio. The numerical method adopted in this work relies on a spectral boundary integral formulation of the elastodynamic relation between the traction stresses acting on the fracture plane located between two linearly elastic half spaces and the resulting displacements. The steps leading to the boundary formulation of this elastodynamic equation are detailed in [46] for the combined spectral formulation and in [47] for the independent spectral formulation. In this article, the 3D independent formulation is adopted, for which the interface tractions $\tau_{j}$ are related to the displacements $u_{j}$ by

$$
\tau_{j}^{ \pm}(x, z, t)=\tau_{0, j}^{ \pm}(x, z, t)-V_{j k} \frac{\partial u_{k}^{ \pm}}{\partial t}(x, z, t)+f_{j}^{ \pm}(x, z, t) .
$$

The first right-hand side (RHS) term $\tau_{0, j}^{ \pm}=\left\{0, \sigma_{0}, 0\right\}^{\top}$ accounts for the preexisting traction stresses present along the interface in absence of any crack. The second RHS term represents the instantaneous response to a change in interface velocity, where $V_{j k}$ is the diagonal matrix

$$
V_{x x}=V_{z z}=\mu / c_{s}, \quad V_{y y}=\eta \mu / c_{s},
$$

with $\mu$ denoting the shear modulus. The last term, $f_{j}^{ \pm}(x, z, t)$, accounts for the history of interface displacements and is expressed in the spectral domain as convolution integrals

$$
\left\{f_{j}^{ \pm}(x, z, t) ; u_{j}^{ \pm}(x, z, t)\right\}=e^{i(k x+m z)}\left\{F_{j}^{ \pm}(k, m, t) ; U_{j}^{ \pm}(k, m, t)\right\}
$$

with

$$
\begin{aligned}
& \left\{\begin{array}{l}
F_{x}^{ \pm}(k, m, t) \\
F_{y}^{ \pm}(k, m, t) \\
F_{z}^{ \pm}(k, m, t)
\end{array}\right\}=-i \mu(2-\eta)\left(\begin{array}{ccc}
0 & -k & 0 \\
k & 0 & m \\
0 & -m & 0
\end{array}\right)\left\{\begin{array}{l}
U_{x}^{ \pm}(k, m, t) \\
U_{y}^{ \pm}(k, m, t) \\
U_{z}^{ \pm}(k, m, t)
\end{array}\right\}-\mu q \int_{0}^{t}\left[i \frac{H_{x y}\left(q c_{s} t^{\prime}\right)}{|q|}\left(\begin{array}{ccc}
0 & -k & 0 \\
k & 0 & m \\
0 & -m & 0
\end{array}\right) \pm H_{y y}\left(q c_{s} t^{\prime}\right)\left(\begin{array}{ccc}
0 & 0 & 0 \\
0 & 0 & 0
\end{array}\right)\right. \\
& \left. \pm \frac{H_{x x}\left(q c_{s} t^{\prime}\right)}{q^{2}}\left(\begin{array}{ccc}
k^{2} & 0 & k m \\
0 & 0 & 0 \\
k m & 0 & m^{2}
\end{array}\right) \pm \frac{H_{z z}\left(q c_{s} t^{\prime}\right)}{q^{2}}\left(\begin{array}{ccc}
m^{2} & 0 & -k m \\
0 & 0 & 0 \\
-k m & 0 & k^{2}
\end{array}\right)\right]\left\{\begin{array}{c}
U_{x}^{ \pm}\left(k, m, t-t^{\prime}\right) \\
U_{y}^{ \pm}\left(k, m, t-t^{\prime}\right) \\
U_{z}^{ \pm}\left(k, m, t-t^{\prime}\right)
\end{array}\right\}|q| c_{s} d t^{\prime}
\end{aligned}
$$




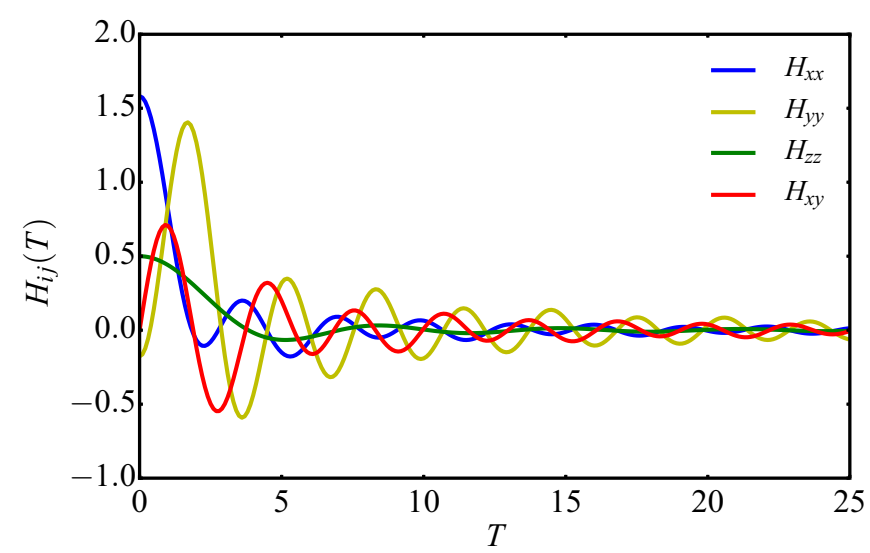

FIG. 1. Convolution kernels $H_{i j}(T)$ for $v=0.35$.

and $q=\sqrt{k^{2}+m^{2}}$. The convolution kernels, $H_{i j}(T)$, whose expressions can be found in [47], are presented in Fig. 1 for the material properties considered in the article $(v=0.35)$. For the mode I fracture problem of interest, the spectral formulation [Eq. (4)] is completed by interface conditions which imply the continuity of tractions and displacements along the interface as long as the normal traction $\tau_{y}$ is lower than the interface strength $\tau^{\text {str }}$ defined in Eq. (1). Otherwise, the fracture process breaks the continuity of displacements and the velocity $\partial u_{j}^{ \pm} / \partial t$ of the crack faces are computed such that $\tau_{y}^{+}=\tau_{y}^{-}=\tau^{\text {str }}$, while the value of the interface strength is related to the opening displacement jump $\delta_{n}=u_{y}^{+}-u_{y}^{-}$ through the cohesive failure model described by Eq. (1). Finally, the elastodynamic relations are integrated in time using an explicit time-stepping scheme

$$
u_{j}^{ \pm}(x, z, t+\Delta t)=u_{j}^{ \pm}(x, z, t)+\frac{\partial u_{j}^{ \pm}}{\partial t}(x, z, t) \Delta t,
$$

with the time step size defined as the fraction $\beta$ of one grid spacing $\Delta x$ traveled by a shear wave: $\Delta t=\beta \Delta x / c_{s}$. In this article, $\beta=0.2$ is chosen to guarantee the stability of the solution as discussed in [47]. The rupture planes studied in this article are typically discretized with $4096 \times 1024$ points in the $x$ and $z$ directions, respectively, providing more than $4 \times 10^{6}$ sampling points along the fracture plane.

\section{METHODOLOGY}

A seed crack whose tips are initially parallel to the $z$ axis is artificially grown in the positive $x$ direction from $x=0$, while the propagation of the left tip is prevented. In an infinite solid under uniform tension $\sigma_{0}$, the rate of energy released by growing a static through crack of size $L$ is given by

$$
G(v=0, L)=\frac{\left(1-v^{2}\right)}{E}\left(\sigma_{0}\right)^{2} \pi \frac{L}{2} .
$$

At $t=0$, the crack reaches the critical size $L_{c}$ where $G\left(v=0, L=L_{c}\right)$ exactly equates the fracture energy $G_{c}^{H}$ and starts to propagate dynamically at a speed $v>0 . L_{c}$ corresponds then to the largest stable crack size $[39,43,48]$ given by

$$
L_{c}=\frac{2 G_{c}^{H}}{\pi\left(\sigma_{0}\right)^{2}} \frac{E}{\left(1-v^{2}\right)},
$$

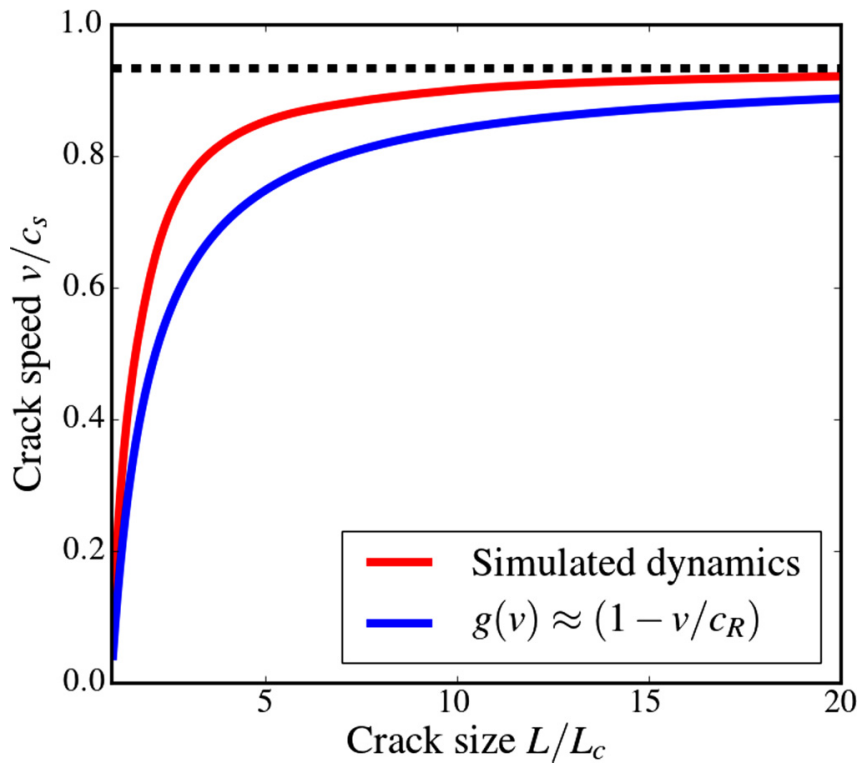

FIG. 2. Typical crack tip dynamics observed along a homogeneous fracture plane. The dashed line highlights the Rayleigh wave speed.

where $E$ and $v$ respectively denote the Young's modulus and Poisson's ratio of the elastic solid, and $G_{c}^{H}$ is the fracture energy of the homogeneous portion of the fracture plane. In an infinite homogeneous solid, Freund [1] showed that the energy release rate evolves with propagation speed as $G(v, L)=$ $g(v) G(v=0, L)$, with $g(v)$ denoting a function which is unity for $v=0$ and zero for $v=c_{R}$. Combining Eqs. (9) and (10), the dynamic energy balance can be expressed as

$$
G(v, L)=G_{c}^{H} \Leftrightarrow \frac{L}{L_{c}}=\frac{1}{g(v)} .
$$

In this framework, Fig. 2 reports the crack dynamics obtained along a perfectly homogeneous interface compared to Freund's approximation for a semi-infinite crack $g(v) \approx(1-$ $v / c_{R}$ ) [1]. In the initial phase of the heterogeneous failure event studied in this article, the crack accelerates with a straight front through a homogeneous region stretching from $x=L_{c}$ to $x=2 L_{c}$, at which point it encounters a tougher circular asperity of diameter $\varnothing=L_{c}$ and fracture energy $G_{c}^{\text {asp }}=$ $\zeta G_{c}^{H}$, with $\tau_{c}^{\text {asp }} / \tau_{c}^{H}=\delta_{c}^{\text {asp }} / \delta_{c}^{H}=\sqrt{\zeta}$. This setup leads to a crack propagation speed $v=0.6 c_{s}$ as the rupture front hits the asperity. Different incident crack front speeds can therefore be investigated by changing the asperity position according to Fig. 2.

Due to the spectral nature of the numerical scheme, which is based on a Fourier series representation of the spatial variation of the interface quantities, the domain of interest is periodic, with a period chosen as $X=10 L_{c}$ and $Z=2.5 L_{c}$. The simulated fracture event thus involves the dynamic interaction of an initially straight mode I crack with a row of circular asperities.

\section{MATERIAL PROPERTIES}

Material properties of Homalite have been chosen for the simulations reported in the article; Young's modulus 


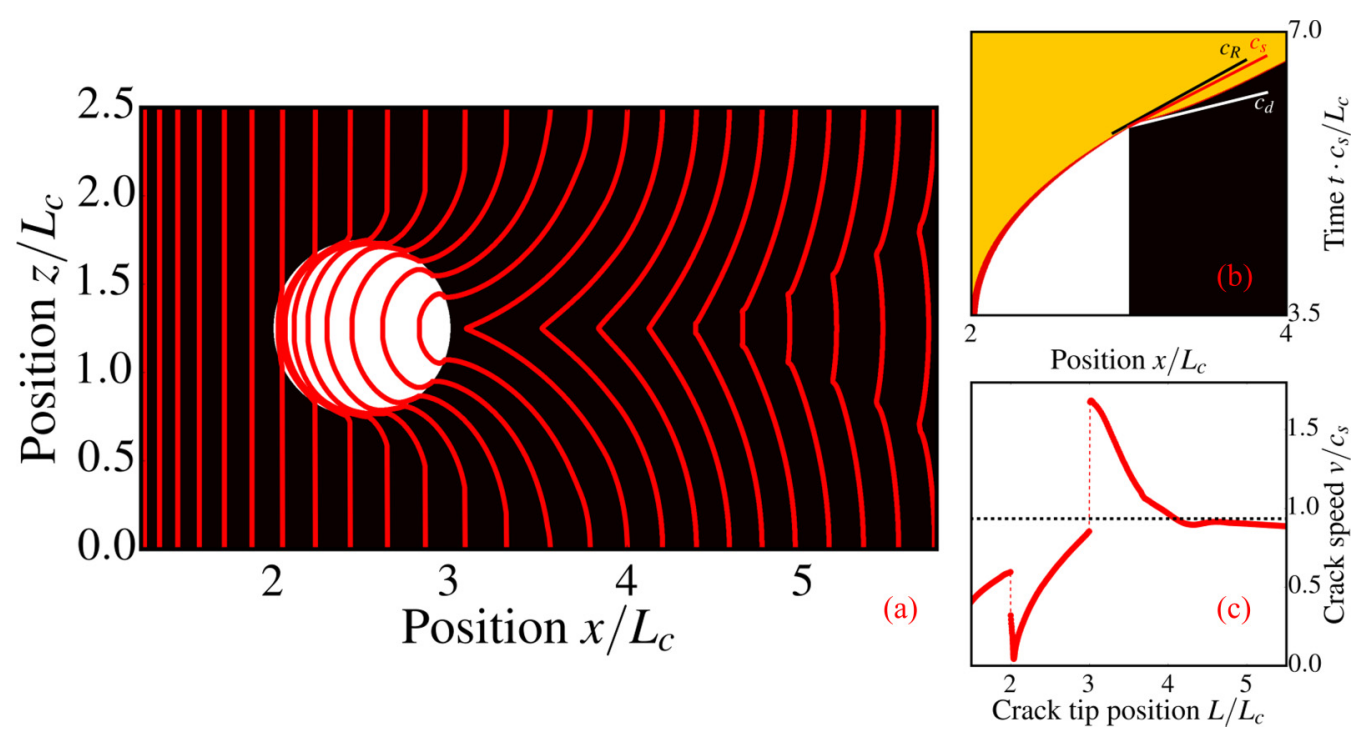

FIG. 3. A supershear burst occurs at the cusp located at the center of the crack front line $z=1.25 L_{c}$. (a) Evolution of the crack front line (in red) captured at regular time interval. (b) Space-time diagram of the rupture event along $z=1.25 L_{c}$. Colors divide broken surface (yellow), cohesive zone (red), and intact interface, which is either black (homogeneous properties) or white (tougher asperity). (c) Evolution of crack front velocity along $z=1.25 L_{c}$. The dashed black line depicts the Rayleigh wave speed.

$E=5.3[\mathrm{GPa}]$, Poisson's ratio $v=0.35$, and shear wave speed $c_{s}=1263[\mathrm{~m} / \mathrm{s}]$. The interface fracture energy $G_{c}^{H}=$ $50\left[\mathrm{~J} / \mathrm{m}^{2}\right]$ is defined by the two parameters entering the cohesive failure model [Eq. (1)] $\tau_{c}^{H}=5[\mathrm{MPa}]$ and $\delta_{c}^{H}=0.02$ $[\mathrm{mm}]$.

\section{SUPERSHEAR BURSTS}

Rice [21] described how the distortion of a crack front interacting with an asperity may locally increase the stress intensity factor to a value sufficiently large to rupture the asperity. Following his first-order perturbation analysis, the interaction of quasistatic [22] and dynamic [23] crack fronts with heterogeneities can be precisely described as long as the perturbation to the crack front is small. Our numerical work thereby aims at widening the investigation toward larger toughness contrasts where higher-order effects cannot be neglected. In this context, our study uncovers the existence of short-lived supershear bursts emerging from large front distortions as presented in Fig. 3 for $\zeta=3$. A parametric study where $\zeta$ is varied can be found later in the article. At the center of the domain (along the line $z=Z / 2=1.25 L_{c}$ ), Fig. 3(c) presents the evolution of crack front speed, which is always in the $x$ direction due to symmetry. Right after rupturing the heterogeneity, the crack speed temporarily exceeds $c_{s}$, which is visually confirmed in Fig. 3(b). This supershear burst extends beyond the center line, as illustrated in Fig. 4(a), which presents the distribution of apparent crack velocity (i.e., the crack velocity $v_{x}$ in the $x$ direction) over the entire crack plane. A related study conducted in mode II [38] has shown that asperities can be triggering sites for supershear propagation of shear cracks. However, unlike its mode II counterpart, super-Rayleigh propagation of tensile (mode I) cracks in a linear elastic material is energetically impossible. This fundamental result of the dynamic theory of LEFM is indeed verified in Fig. 4(b), which presents the spatial distri- bution of the crack speed $v_{n}$ computed normal to the front. As apparent there, the crack speed remains sub-Rayleigh as long as the crack front curvature is continuous. The evolution of the crack front shape presented in Fig. 3(a) reveals how the emergence of a cusp along the front line coincides with the episode of supershear propagation. The latter emerges
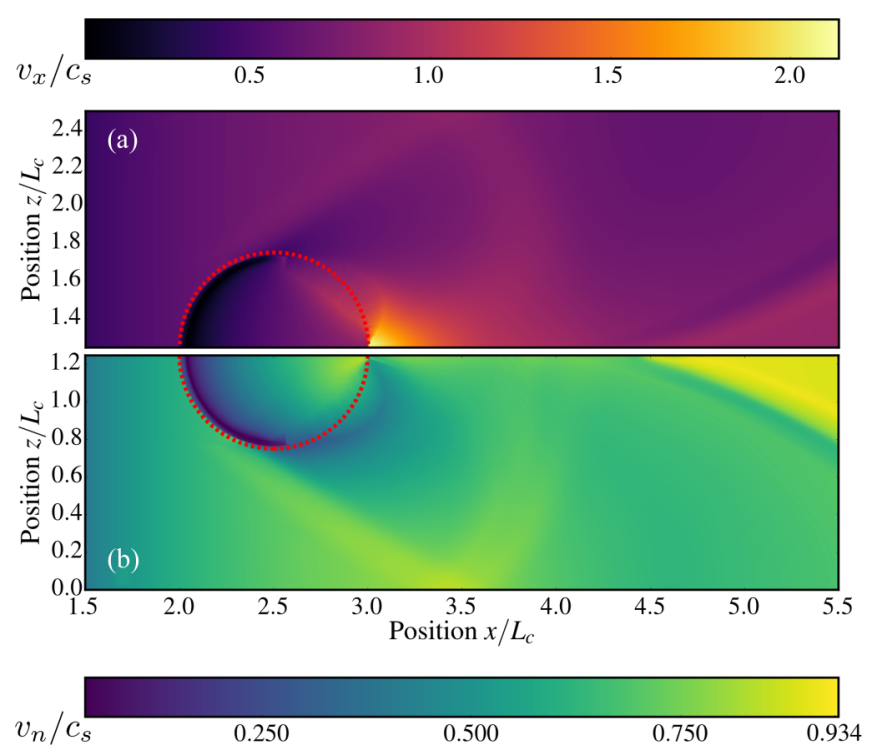

FIG. 4. The supershear burst arises exclusively at the cusp emerging along the front at $z=Z / 2$. Evolution of the crack front velocity $v_{x} / c_{s}$ in the $x$ direction (a) and in the direction normal to the crack front $v_{n} / c_{s}$ (b) computed along the interface for $z<Z / 2$ and $z>Z / 2$. The color map is scaled between the minimum and maximum values verifying the supershear range of the apparent forward velocity $v_{x}$ and the sub-Rayleigh range of the normal crack speed $v_{n}\left(c_{R} \cong 0.934 c_{s}\right)$ predicted by LEFM for a smooth crack front. 


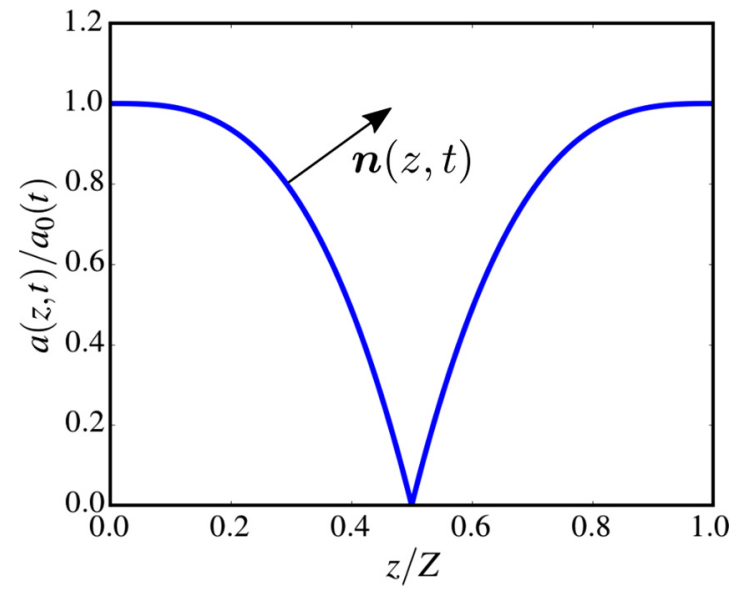

FIG. 5. Schematic representation of a kinked crack front, with $\boldsymbol{n}$ denoting the unit normal at $(z, t)$.

exclusively at $z=Z / 2$ where the symmetry of the problem implies that $v_{n}=v_{x}$, except in the presence of a cusp for which the normal is not defined and LEFM assumptions break down. Nevertheless, the propagation velocity of the cusp can be rationalized with geometric arguments, as presented in the next section.

\section{GEOMETRICAL MODEL}

Next, we introduce a geometrical model that explains and predicts how a sub-Rayleigh crack velocity in the normal direction of the front can yield supershear velocity on the projected forward direction, when a geometrical cusp is formed.

Let $a(z, t)$ denote the amplitude of the front distortion, i.e., the advance of the crack front at location $z$ and time $t$ relative to the front location at the kink $(z=Z / 2)$ (Fig. 5). Let us assume that the crack front propagates at uniform subsonic speed $\kappa c_{R}$ (with $\kappa<1$ ) in the direction of the local normal $\boldsymbol{n}$ to the front. Geometrical arguments readily lead to the following approximate expression of the forward velocity $v_{x}$ of the front:

$$
\frac{v_{x}(z, t)}{c_{R}} \approx \kappa \sqrt{1+\left(\frac{\partial a(z, t)}{\partial z}\right)^{2}} .
$$

Based on the observed shape of the front immediately past the asperity (see Fig. 3), let us adopt the following expression for the shape of the front between $0 \leqslant z \leqslant Z / 2$ :

$$
a(z, t)=a_{0}(t)\left[-8(z / Z)^{3}+1\right],
$$

where $a_{0}(t)=a(0, t)=a(Z, t)$. Equation (13) corresponds to the lowest-order polynomial satisfying $a(0, t)=a_{0}(t)$, $a(Z / 2, t)=0$ as well as the continuity of tangents and curvatures across the periodic boundaries, i.e., $\frac{\partial a}{\partial z}(0, t)=$ $\frac{\partial^{2} a}{\partial z^{2}}(0, t)=0$. Combining Eqs. (12) and (13), we obtain the values of the front perturbation at which the center point $(z=Z / 2)$ is predicted to propagate faster than $c_{R}$ :

$$
\frac{a_{0}}{Z}=\frac{1}{6 \kappa} \sqrt{1-\kappa^{2}} .
$$

This relation is presented in Fig. 6. As expected, the amplitude of the front perturbation $a_{0}$ at which supershear crack motion

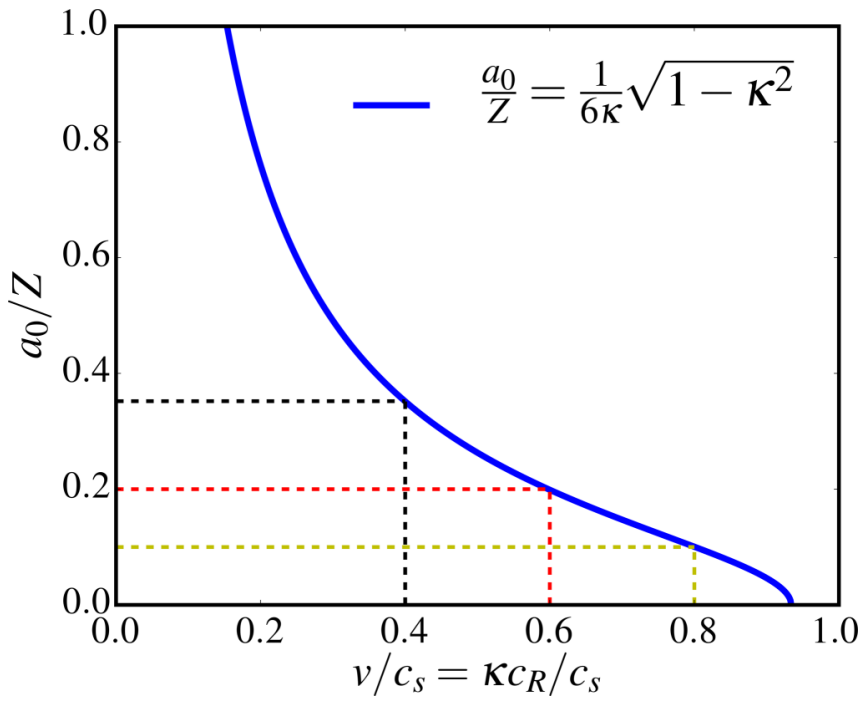

FIG. 6. Amplitude of the front perturbation required to observe $v_{x}>c_{R}$ at the center point as a function of the incident crack speed $v$. The dashed lines highlight the required amplitude for each of the three different crack speeds considered in the parametric study presented in Fig. 8.

appears decreases with increasing normal crack speed (i.e., with increasing value of $\kappa$ ).

For a given amplitude of the perturbation $a_{0} / Z$, we can also compute the section of the crack front defined as $z^{*} \leqslant z \leqslant$ $\left(Z-z^{*}\right)$ that has a forward motion faster than the Rayleigh wave speed.

$$
\frac{z^{*}}{Z}=\left(\frac{\sqrt{1-\kappa^{2}}}{24 \kappa\left(a_{0} / Z\right)}\right)^{1 / 2} .
$$

This relation is shown in Fig. 7 for different values of the normal crack front speed. For the reference case of the article

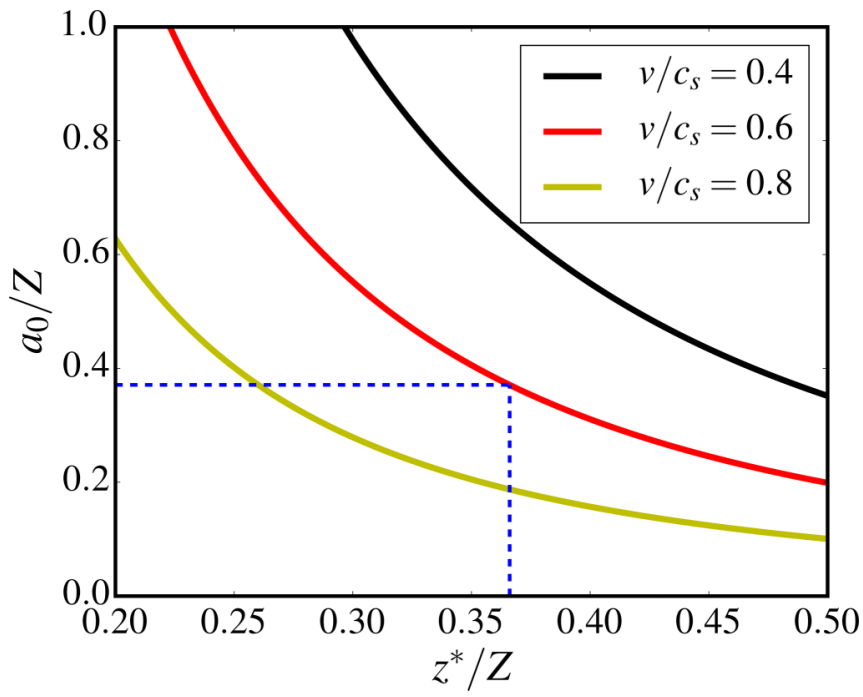

FIG. 7. Portion of the crack front moving with a forward velocity $v_{x}>c_{R}$. The dashed blue line emphasizes the expected portion for the reference case studied in Fig. 4, while the value $a_{0} / Z=0.371$ is read from Fig. $8\left(v=0.6 c_{s}\right.$ and $\left.\zeta=3\right)$. 


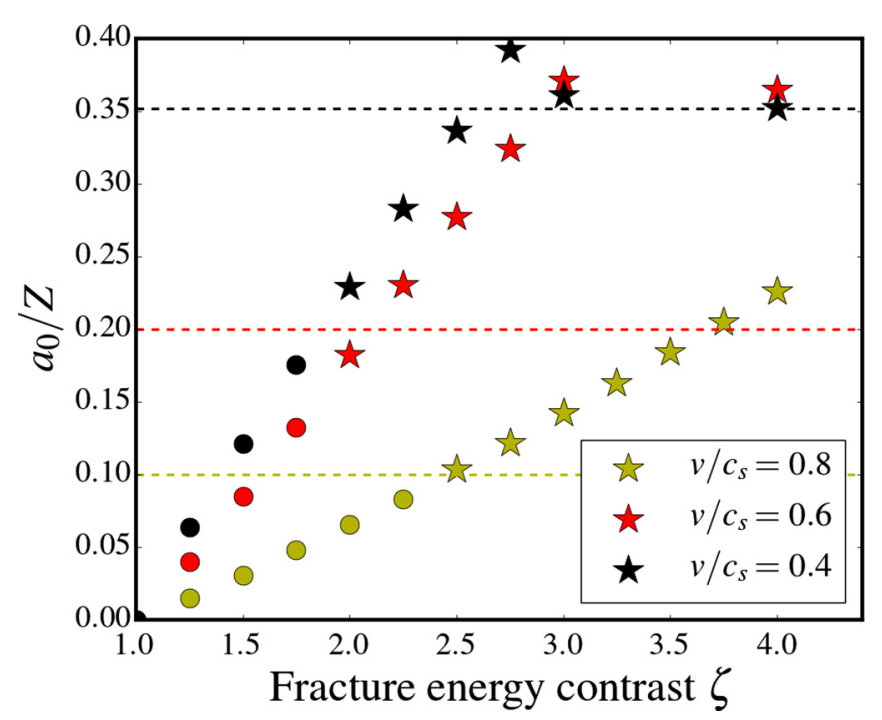

FIG. 8. Maximum crack front deflection $a_{0}$ observed for various asperity toughnesses and three different values of the incident crack speed $v / c_{s}$. The deflection of the crack front interacting with the tougher asperity either creates a supershear burst (star symbols) or not (round symbols). From the geometrical model [cf. Eq. (14) and Fig. 6], this observed transition is predicted to occur at a given crack front deflection, which depends on the incident crack speed and is depicted by the horizontal dashed lines.

$\left(v=0.6 c_{s}\right)$, Eq. (15) predicts therefore that the crack front, just after being released from the asperity, has an apparent forward velocity $v_{x}>c_{R}$ along a portion corresponding to $0.37 \leqslant z / Z \leqslant 0.63$ (or equivalently $0.925 \leqslant z / L_{c} \leqslant 1.575$ ) in agreement with the velocity profile presented in Fig. 3(a).

\section{PARAMETRIC STUDY}

The supershear bursts studied in the article for $v=0.6 c_{s}$ and $\zeta=3.0$ are also observed with different incident crack speeds or asperity toughnesses as reported in Fig. 8. The dashed lines correspond to the predicted minimum front perturbation required to observe a supershear burst at the center of the domain according to Eq. (14). The proposed model gives therefore a quantitatively good prediction with fast crack front speeds. At slower crack velocity, the front perturbation becomes larger and stops complying with the model hypothesis of a uniform speed in the normal direction. This inverse relationship between the incident rupture speed and the front deflection is obvious in Fig. 8 and can be understood from Freund's crack tip equation of motion [1]:

$$
G_{c}=G=\frac{\left(1-v^{2}\right)}{E} A_{I}(v) K_{I}^{2} .
$$

In Eq. (16), $K_{I}$ and $A_{I}(v)$ are respectively the mode I stress intensity factor and universal function defined by

$$
A_{I}(v)=\frac{\alpha_{d} v^{2}}{(1-v) D c_{s}^{2}},
$$

where $\alpha_{s, d}^{2}=1-v^{2} / c_{s, d}^{2}$ and $D=4 \alpha_{d} \alpha_{s}-\left(1+\alpha_{s}^{2}\right)^{2}$. The front perturbation analyses presented in [21-23] describe how the crack front deflection created by the presence of the asperity leads to an increase of the stress intensity factor $\Delta K_{I}$ at the edge of a tougher heterogeneity to compensate its associated increase in interface fracture energy $\Delta G_{c}$. In a first-order approximation, this change in energy release rate can be written as

$$
G_{c}+\Delta G_{c}=\frac{\left(1-v^{2}\right)}{E} A_{I}(v)\left(K_{I}+\Delta K_{I}\right)^{2} .
$$

The velocity-dependent coefficient $A_{I}(v)$ monotonically increases with $v$, which implies that the interaction of a faster crack speed with a given heterogeneity characterized by $\Delta G_{c}$ leads to a lower $\Delta K_{I}$, i.e., to a smaller deflection of the crack front. For a more detailed description of the link between the perturbation amplitude for a dynamically propagating crack front and the associated effect on the local value of the stress intensity factor, please see [23].

\section{SHOCK WAVES}

Our study demonstrates how supershear propagation can also exist in the tensile failure of linear elastic materials. However, supershear events emerge exclusively where the crack front curvature is discontinuous and occur thereby in the form of localized bursts along the crack front. Nevertheless, these short-lived bursts can have a significant impact on the overall rupture dynamics through the creation of shock waves associated with these supershear propagation events. Figure 9(a) presents the profile of the normal opening velocity field $\dot{\delta}_{n}$ just after the rupture of the heterogeneity. Two surface wave fronts can be identified: a circular front released by the failure of the asperity and growing radially at the Rayleigh wave speed along the fracture surface, and a triangular front characteristic of the shock wave generated by the supershear motion of the crack. For several locations along the fracture plane, Fig. 9(b) shows how the amplitudes of these wave fronts are comparable to the opening velocities observed during the rupture. Moreover, as the circular wave front progressively decays as it expands along the fracture surface, the triangular shock wave front propagates along the fracture surface with a persistent amplitude (Rayleigh Mach front [35]), as highlighted in Fig. 9(c). The shock waves visible in Fig. 9 are moving at the Rayleigh wave speed and are hence progressively catching up with the sub-Rayleigh rupture front. A video of this rupture event is provided as Supplemental Material [49]. As extensively discussed in the context of mode II cracks [7,35,50], this supershear event should also radiate a shear Mach cone out of the interface but the latter is not directly observable using the boundary integral formulation of interest.

\section{CONCLUSION}

Taking advantage of the fine discretization allowed by the numerical scheme based on a spectral boundary integral formulation of the 3D elastodynamic equations, we study the large distortion of an initially straight dynamically propagating crack front as it interacts with a circular asperity. Our study uncovers the existence of supershear bursts emerging during the tensile failure of a linearly elastic material, beyond the range of crack propagation speeds allowed by the classical 


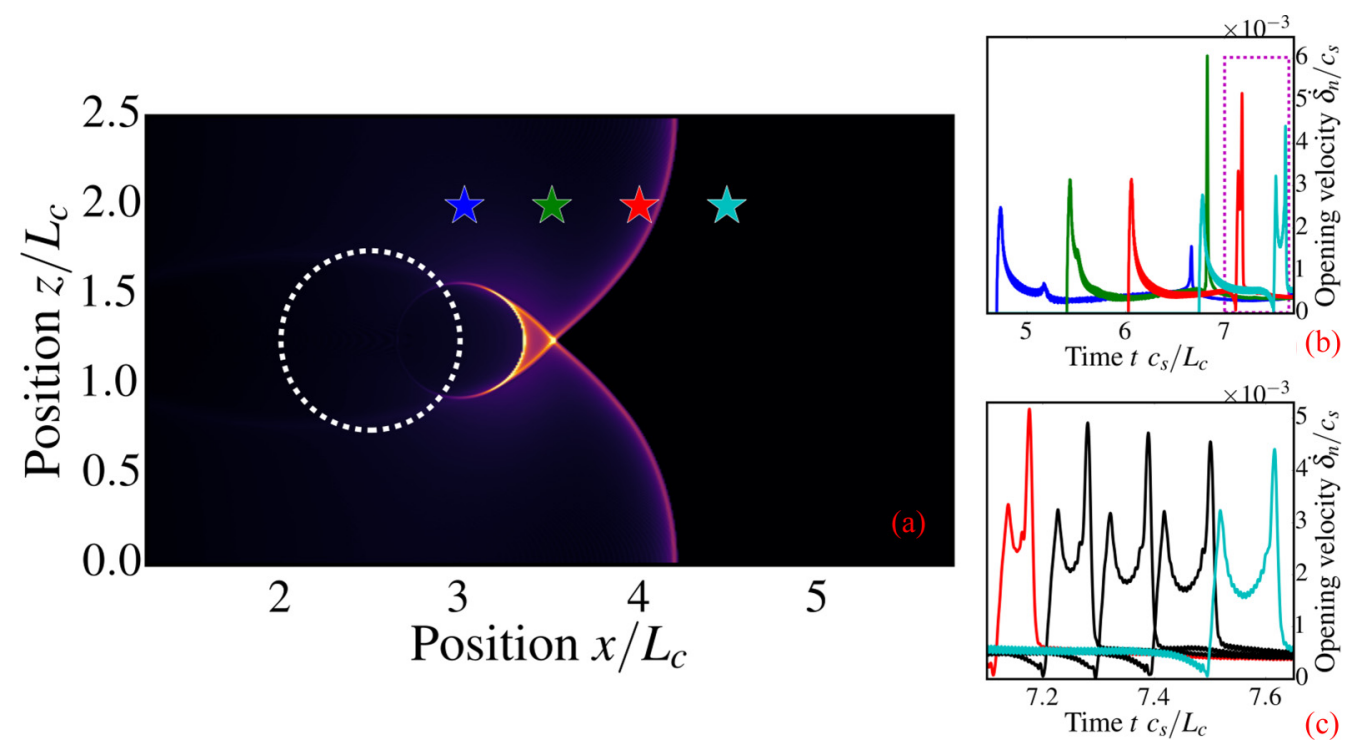

FIG. 9. The supershear burst creates shock waves driving energy far from the asperity site. (a) Snapshot of crack opening velocity field $\dot{\delta}_{n}$ following the failure of the asperity whose position is highlighted by the white dashed circle. The colored stars denote the positions at which the time evolution of $\dot{\delta}_{n}$ is computed and presented in (b) and (c). (b) Evolution of $\dot{\delta}_{n}$ observed at the different positions highlighted in (a). The strong surface waves caused by the rupture of the asperity are visible after the initial peak characteristic of the rupture front. (c) Zoom-in of $\dot{\delta}_{n}$ history [dotted rectangle in (b)] emphasizing first the trace left by the persistent "triangular" shock wave, sharply followed by the decaying "circular" front. The black curves correspond to additional sampling points located between the red and cyan positions.

theory of dynamic LEFM. The crack front speeds computed in a direction normal to the propagating front remain subRayleigh as long as the front curvature is continuous, and the supershear bursts are associated with the formation of a cusp in the crack front caused by the heterogeneity. These intersonic episodes create shock waves persisting along the fracture surfaces far from the asperity site. Several experimental studies $[5,6,13]$ reported how fast tensile ruptures (between a few tenths of $c_{s}$ and the branching velocity) diverge from LEFM predictions. These events are characterized by significant crack front distortions caused by macro- [51] and microscopic $[8,14]$ heterogeneities and/or the nucleation of microbranches able to form cusps along the crack front line
[26]. In this context, the resulting discontinuities are favorable sites to trigger these short-lived "sonic booms" capable of significantly impacting the overall rupture dynamics. We hope that our work will motivate further investigations on heterogeneous dynamic fracture to establish whether experimental ruptures confirm our linear elastic predictions or if material nonlinearities and/or viscosity impede the emergence of supershear bursts.

\section{ACKNOWLEDGMENT}

This work was supported by the Swiss National Science Foundation (Grant No. 162569).
[1] L. B. Freund, Dynamic Fracture Mechanics, Cambridge Monographs on Mechanics and Applied Mathematics (Cambridge University Press, Cambridge, 1990).

[2] B. V. Kostrov and S. Das, Principles of Earthquake Source Mechanics, Cambridge Monographs on Mechanics and Applied Mathematics (Cambridge University Press, Cambridge, 1988).

[3] R. Burridge, Geophys. J. Int. 35, 439 (1973).

[4] D. J. Andrews, J. Geophys. Res. 81, 5679 (1976).

[5] K. Ravi-Chandar and W. G. Knauss, Int. J. Fract. 26, 65 (1984).

[6] J. Fineberg and M. Marder, Phys. Rep. 313, 1 (1999).

[7] A. J. Rosakis, Adv. Phys. 51, 1189 (2002).

[8] K. Ravi-Chandar and W. G. Knauss, Int. J. Fract. 26, 141 (1984).

[9] K. Ravi-Chandar and W. G. Knauss, Int. J. Fract. 25, 247 (1984).

[10] E. Bouchbinder, T. Goldman, and J. Fineberg, Rep. Prog. Phys. 77, 046501 (2014).
[11] J. Fineberg and E. Bouchbinder, Int. J. Fract. 196, 33 (2015).

[12] E. Sharon and J. Fineberg, Nature (London) 397, 333 (1999).

[13] J. Scheibert, C. Guerra, F. Célarié, D. Dalmas, and D. Bonamy, Phys. Rev. Lett. 104, 045501 (2010).

[14] C. Guerra, J. Scheibert, D. Bonamy, and D. Dalmas, Proc. Natl. Acad. Sci. USA 109, 390 (2012).

[15] T. G. Boué, G. Cohen, and J. Fineberg, Phys. Rev. Lett. 114, 054301 (2015).

[16] P. D. Washabaugh and W. G. Knauss, Int. J. Fract. 65, 97 (1994).

[17] A. J. Rosakis, O. Samudrala, and D. Coker, Science 284, 1337 (1999).

[18] A. Livne, O. Ben-David, and J. Fineberg, Phys. Rev. Lett. 98, 124301 (2007).

[19] T. Goldman, R. Harpaz, E. Bouchbinder, and J. Fineberg, Phys. Rev. Lett. 108, 104303 (2012).

[20] J. Bleyer and J.-F. Molinari, Appl. Phys. Lett. 110, 151903 (2017). 
[21] J. R. Rice, J. Appl. Mech. 52, 571 (1985).

[22] H. Gao and J. R. Rice, J. Appl. Mech. 56, 828 (1989).

[23] J. R. Willis and A. B. Movchan, J. Mech. Phys. Solids 43, 319 (1995).

[24] D. Bonamy and E. Bouchaud, Phys. Rep. 498, 1 (2011).

[25] L. Ponson, Int. J. Fract. 201, 11 (2016).

[26] I. Kolvin, G. Cohen, and J. Fineberg, Phys. Rev. Lett. 114, 175501 (2015).

[27] R. J. Archuleta, J. Geophys. Res.: Solid Earth 89, 4559 (1984).

[28] M. Bouchon, M.-P. Bouin, H. Karabulut, M. N. Toksöz, M. Dietrich, and A. J. Rosakis, Geophys. Res. Lett. 28, 2723 (2001).

[29] M. Bouchon, Science 301, 824 (2003).

[30] E. M. Dunham, Bull. Seismol. Soc. Am. 94, S256 (2004).

[31] H. Yue, T. Lay, J. T. Freymueller, K. Ding, L. Rivera, N. A. Ruppert, and K. D. Koper, J. Geophys. Res.: Solid Earth 118, 5903 (2013).

[32] K. Xia, A. J. Rosakis, and H. Kanamori, Science 303, 1859 (2004).

[33] K. Xia, A. J. Rosakis, H. Kanamori, and J. R. Rice, Science 308, 681 (2005).

[34] X. Lu, N. Lapusta, and A. J. Rosakis, Proc. Natl. Acad. Sci. USA 104, 18931 (2007).

[35] E. M. Dunham and H. S. Bhat, J. Geophys. Res.: Solid Earth 113 (2008).

[36] I. Svetlizky, D. Pino Munoz, M. Radiguet, D. S. Kammer, J.-F. Molinari, and J. Fineberg, Proc. Natl. Acad. Sci. USA 113, 542 (2016).

[37] E. Fukuyama and K. B. Olsen, Pure Appl. Geophys. 159, 2047 (2002).
[38] E. M. Dunham, P. Favreau, and J. M. Carlson, Science 299, 1557 (2003).

[39] Y. Liu and N. Lapusta, J. Mech. Phys. Solids 56, 25 (2008).

[40] F. Barras, D. S. Kammer, P. H. Geubelle, and J.-F. Molinari, Int. J. Fract. 189, 149 (2014).

[41] X. Ma and A. Elbanna, Geophys. J. Int. 203, 664 (2015).

[42] G. Albertini and D. S. Kammer, J. Geophys. Res.: Solid Earth 122, 6625 (2017).

[43] F. Barras, P. H. Geubelle, and J.-F. Molinari, Phys. Rev. Lett. 119, 144101 (2017).

[44] F. X. Passelegue, A. Schubnel, S. Nielsen, H. S. Bhat, and R. Madariaga, Science 340, 1208 (2013).

[45] E. Sharon, S. P. Gross, and J. Fineberg, Phys. Rev. Lett. 76, 2117 (1996).

[46] P. H. Geubelle and J. R. Rice, J. Mech. Phys. Solids 43, 1791 (1995).

[47] M. S. Breitenfeld and P. H. Geubelle, Int. J. Fract. 93, 13 (1998).

[48] T. L. Anderson, Fracture Mechanics: Fundamentals and Applications, 3rd ed. (Taylor \& Francis, Boca Raton, FL, 2005).

[49] See Supplemental Material at http://link.aps.org/supplemental/ 10.1103/PhysRevE.98.063002 for a video of the rupture event studied in the article. The evolution of the fracture process is presented (top frames) with the same color code as in Fig. 3(b), while the evolution of the crack opening velocity is shown (bottom frames) using the same color code and scale as in Fig. 9(a).

[50] M. Mello, H. S. Bhat, and A. J. Rosakis, J. Mech. Phys. Solids 93, 153 (2016).

[51] F. Kerkhof, in Proceedings of an International Conference on Dynamic Crack Propagation, edited by G. C. Sih (Springer, Dordrecht, 1973), pp. 3-35. 